\title{
LC3 and ATG5 overexpression and neuronal cell death in the prefrontal cortex of postmortem chronic methamphetamine users
}

\author{
Shahrokh Khoshsirat ${ }^{a}$ Maryam Sadat Khoramgah ${ }^{\text {ab }}$ Gholam-Reza Mahmoudiasl $^{\text {b }}$ Mostafa \\ Rezaei-Tavirani ${ }^{\mathrm{c}}$ Mohammad Amin Abdollahifar ${ }^{\mathrm{d}}$ Foozhan Tahmasebinia $^{\text {af }}$ Shahram Darabi ${ }^{\mathrm{e}}$ \\ Somayeh Niknazar ${ }^{\mathrm{a}}$ Hojjat Allah Abbaszadeh ${ }^{\text {ab }}$ \\ Hearing Disorders Research Center, Loghman Hakim Hospital, Shahid Beheshti University of \\ Medical Sciences, Tehran, Iran \\ b \\ Laser Application in Medical Sciences Research Center, Shahid Beheshti University of Medical \\ Sciences, Tehran, Iran \\ Proteomics Research Center, Faculty of Para Medicine, Shahid Beheshti University of Medical \\ Sciences, Tehran, Iran \\ Department of Biology and Anatomy, School of Medicine, Shahid Beheshti University of Medical \\ Sciences, Tehran, Iran \\ Cellular and Molecular Research Center, Qazvin University of Medical Sciences, Qazvin, Iran \\ Department of Biological Sciences, Southern Methodist University, Dallas, Texas, USA
}

\begin{abstract}
Methamphetamine (METH) abuse is accompanied by oxidative stress, METH-induced neurotoxicity, and apoptosis. Oxidative stress has devastating effects on the structure of proteins and cells. Autophagy is an evolutionarily conserved intracellular regulated mechanism for orderly degradation of dysfunctional proteins or removing damaged organelles. The precise role of autophagy in oxidative stress-induced apoptosis of dopaminergic neuronal cells caused by METH has not clarified completely. In this study, we sought to evaluate the effects of METH abuse on autophagy in the prefrontal cortex of postmortem users, mainly focusing on the ATG5 and LC3 during neuroinflammation. Postmortem molecular and histological examination was done for two groups containing 12 non-addicted and 14 METH addicted cases. ATG5 and LC3 expression were analyzed by real-time PCR and immunohistochemistry (IHC) methods. Histopathological analysis was performed by stereological cell counting of neuronal cells using Hematoxylin and Eosin (H \& E) staining technique. In order to detect DNA damage in the prefrontal lobe, Tunnel staining was performed. Real-time PCR and IHC assay showed overexpression of ATG5 and LC3 protein in the prefrontal cortex of Meth users. The cell death and neuronal degeneration were increased significantly based on Tunel assay and the stereological analysis in the Prefrontal cortex. Chronic METH exposure probably induces ATG5 and LC3 overexpression and neuronal cell death in the Prefrontal cortex of the postmortem cases.
\end{abstract}

Keywords: ATG5; Cell death; LC3; Methamphetamine; Prefrontal cortex. 